\title{
Boost Up Quality Assurance through Regular Evaluation of Proficiency Testing Performance
}

\author{
Rajendra Dev Bhatt ${ }^{1 *}$, Dipesh Tamrakar ${ }^{1}$, Bishal Maharjan ${ }^{1}$, Rajendra Duwal ${ }^{1}$, Raj Kumar Shrestha ${ }^{1}$, Surendra Koju ${ }^{2}$, \\ Prabodh Risal ${ }^{1}$
}

\begin{abstract}
Affiliations:
${ }^{1}$ Department of Biochemistry, Kathmandu University School of Medical Sciences, Dhulikhel, Kavre, Nepal

${ }^{2}$ Department of Pathology, Kathmandu University School of Medical Sciences, Dhulikhel, Kavre, Nepal
\end{abstract}

\section{Correspondence to:}

Rajendra Dev Bhatt

Department of Biochemistry

Kathmandu University School of Medical Sciences

Dhulikhel Hospital, Dhulikhel, Kavre, Nepal

bhattdev.rajendra@gmail.com

\section{How to cite this Article:}

Bhatta D. R., Tamrakar D., Maharjan B., Duwal R., Shrestha RK, Koju S, Risal P. Boost Up Quality Assurance through Regular Evaluation of Proficiency Testing Performance. Ann. Clin. Chem. Lab. Med. 2017:3(1); 3-7

DOI: http://dx.doi.org/10.3126/acclm.v3i1.17296

\section{(C) 2017 Nepalese Association for Clinical Chemistry}

\begin{abstract}
BACKGROUND

Participation in External Quality Assessment (EQA) program with practice of Internal Quality Control (IQC) is the excellent indicator of good laboratory practice (GLP) to assure quality services. EQA/proficiency testing is an essential component of Quality Assurance to assess the analytical performance compared within same methods of other laboratories. It is also important to detect equipments failure, identifying reagent problems, reviewing staff training as well as to initiate and evaluate corrective action timely.
\end{abstract}

\section{METHODS}

This is descriptive analysis of the feedback received from EQAS providing institute for twenty routine biochemical parameters which are most commonly performed in the Clinical Biochemistry Laboratory of Dhulikhel Hospital. Variation Index Score (VIS), Overall Mean VIS (OMVIS) and Standard Deviation Index (SDI) of all parameters for the year 2014 and 2015 were analyzed statistically.

\section{RESULTS}

On the analysis of all the routine biochemical parameters regularly participated in EQAS, over all mean variation index score (OMVIS) of individual tests found 55\%, 30\% and 15\% under good, very good and satisfactory categories respectively.

\section{CONCLUSION}

Participation and periodic evaluation of EQAS indicators along with internal quality control practice has been galvanizing good laboratory practice and scientifically boosts up quality service in clinical laboratories.

KEY WORDS: External Quality Assessment Scheme (EQAS), Internal Quality Control (IQC), Good Laboratory Practice (GLP), Overall Mean Variation Index Score (OMVIS). 


\section{INTRODUCTION}

Clinical laboratories play vital role in diagnosis, management and follow-up of patients. Appropriate quality management system is therefore necessary. All the Medical laboratories must have internal quality control and should participate in external quality assessment (EQA) programs/proficiency testing (PT) to assure their patients and clinicians ${ }^{1}$.Quality control is one of the most scientific and significant tool to correct errors in analytical phase in the clinical laboratories. External quality assessment (EQA) and internal quality control (IQC) are critical part of good laboratory practice (GLP) to ensure quality reports. IQC is self-assessing tool by using control materials for the continuous and immediate monitoring of the analytical part.

Those clinical laboratories wishing to improve their quality standards and provide the best possible results, they must participate in an External Quality Assessment Scheme(EQAS) ${ }^{2}$. For a proficiency test, a series of unknown samples are sent to the laboratory from the program offering institute. The samples are analyzed in the same manner as patient specimens, and the results are reported to the proficiency testing body. The program then compiles the results from all of the participating laboratories in the survey and sends a performance report back to each participating laboratory.EQA is an essential indicator of Quality Assurance to assess the analytical performance compared within same methods of other laboratories ${ }^{3}$. It is also important to detect equipment failure, identifying reagent problems, reviewing staff training as well as to initiate and evaluate corrective action timely ${ }^{4}$, this means it is crucial for pre and post analytical phase too.

Clinical Biochemistry Laboratory of Dhulikhel Hospital-Kathmandu University Hospital (DH-KUH) has been participating in External Quality Assessment Scheme (EQAS) of Christian Medical College (CMC), Vellore, India under the aegis of Association of Clinical Biochemist of India. As a participating laboratory, department of clinical biochemistry tried to improve laboratory services by regular practice of quality control of reagents used, test kits, procedures and equipment since last decade. The participation only is not sufficient so regular analysis of quality indicator received from EQAS governing body is equally important.

\section{METHODS}

Clinical Biochemistry Department of Dhulikhel Hospital-Kathmandu University Hospital has been receiving EQAS samples from Christian Medical College (CMC), Vellore, India since 2004. EQAS reports submitted in web database from January 2014 to December 2015 were taken in this study. The most frequent test parameters were analyzed in term of EQAS statistical like Variation Index Score (VIS), Overall Mean Variation Index Score (OMVIS) and Standard Deviation Index (SDI) as following formula.

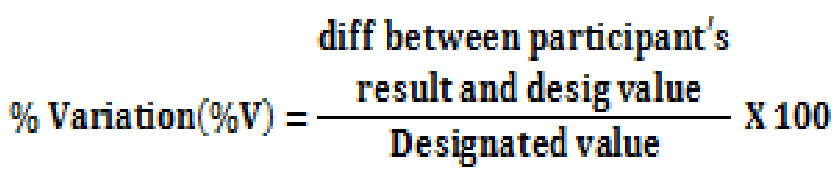

Variance Index(VIS) $=\frac{\% \text { Variation }}{\text { Desired CV }} \times 100$

On the day of EQA sample analysis aliquot was prepared according to guidelines from lyophilized samples provided by EQAS providing body. Generally, EQA samples were analyzed in second week of every month because before 20 of respected month all obtained value must be submitted. Enrolled tests were performed from that aliquot exactly as patient's sample and observed values were documented and entered web based database of EQAS provider by clinical biochemist or authorized medical technologist. 
Observed values of EQA samples were not allowed to submit after 20 of any months. But in case of delay in EQA sample delivery from EQAS provider, in such situation they consider observed values before the end of months by email.

\section{RESULTS}

While comparing VIS for all specific parameters there is no significant difference observed in the year 2014 and 2015. Every month, on average, $17 \%$ results were in not acceptable range according to VIS. However, there is no single test result found whose VIS is more than 200 continuously for three months.

On the analysis of 20 most common biochemical parameters in the year 2014, individual test wise OMVIS, 55\% (11 parameters) of total EQAS enrolled parameters were under good, followed by $30 \%$ (6 parameters) in very good and $15 \%$ (3 parameters) in satisfactory groups. None of any parameters fall under not acceptable category. The OMVIS in the year 2015 for the same parameter is $30 \%$ very good, $45 \%$ good and $25 \%$ satisfactory.

On the evaluation of monthly OMVIS distribution, similar pattern of performance was observed for both years. with improvement for first seven months whereas reduced performance was noticed in last five months period of the year 2015.There is no

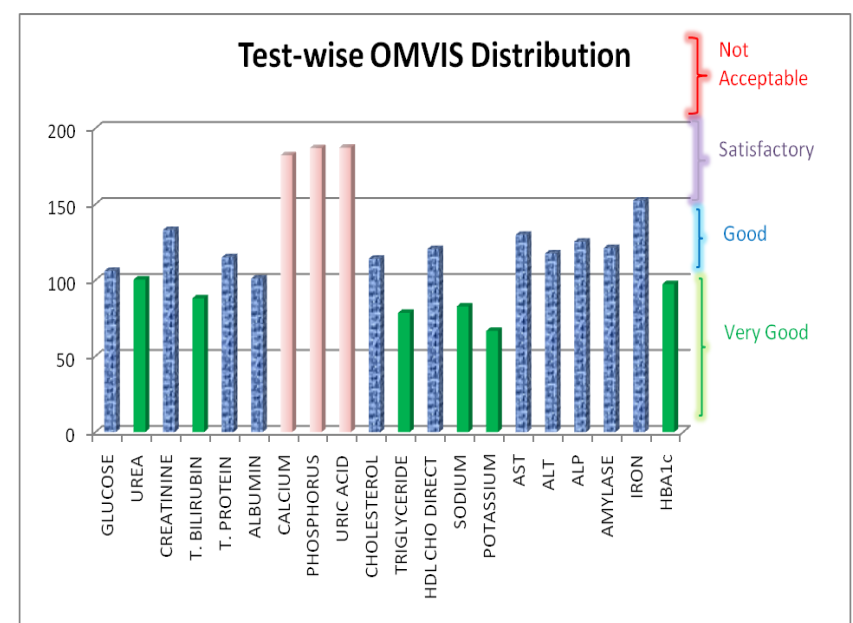

Figure 1: Test-wise OMVIS

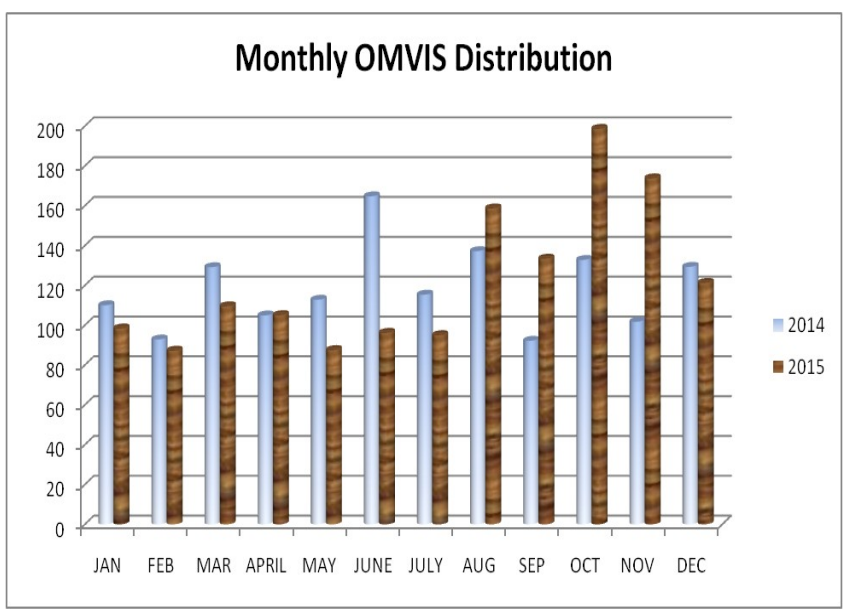

Figure 2: Monthly OMVIS

significant difference found in monthly OMVIS 2014 and 2015.

Significant increase in rank among participant laboratories were observed on 7 test parameters, in which dramatic progress was seen in electrolyte $\left(\mathrm{Na}^{+}\right.$and $\left.\mathrm{K}^{+}\right)$. From 2014 to 2015 , the position of this laboratory for $\mathrm{Na}^{+}$ and $\mathrm{K}^{+}$were 539 to 74 out of 2635 and 256 to 23 out of 2656 respectively.

\section{DISCUSSION}

Participation with dedication in EQAS and regular analysis of feedback provided by EQA / Proficiency testing body is a crucial indicator of quality assurance to monitor and correct the laboratory performance(5). VIS of various biochemical parameters attracts the eyes of

responsible authorities of clinical laboratories to specify the deviations from the likely result. When there is significant deviation, a participation laboratory should take curative action in needed area like, quality of reagents in all aspects, instruments, implementation of standard operating procedure and training of human resources. OMVIS of 2015 showed significant improvement compare to 2014 especially in first seven months. However last five months of year 2015 has high OMVIS in respective months of year 2014, this might be due to poor storage and transportation of reagents and/or control materials during border blockade after devastating earthquake in Nepal. The percentage of not acceptable results measured in first seven months of 2015 was 
only $7 \%$, which was $17 \%$ in same months of year 2014 .

Frequency of high VIS was especially encountered in the results of calcium, phosphorous and uric acid and this is most probably due to the poor quality of deionized water and single arm clinical chemistry analyzer. Department has decided to install their own deionized water plant and a new multi arm clinical chemistry analyzer to improve this matter. High VIS for uric acid is most probably due to the carryover and deterioration of reagents on board of analyzer, similar problem was found in (Yadav $\mathrm{R}$, et al) study ${ }^{6}$.This is the most beautiful aspect of proficiency testing which force the clinical laboratories to change something, especially wrong ones $^{7,8}$.

Mean SDI of nineteen parameters was inacceptable range $(<1.25)$ while serum iron test mean SDI was in marginal performance. Department has changed the then used iron reagents and method followed. This change is made possible only due to the analysis of proficiency testing performance. Significant increase in rank among participant laboratories were observed on 7 test parameters, in which dramatic progress was seen in electrolyte $\left(\mathrm{Na}^{+}\right.$and $\mathrm{K}^{+}$). From 2014 to 2015 , the position of this laboratory for $\mathrm{Na}^{+}$and $\mathrm{K}^{+}$were 539 to 74 out of 2635 and 256 to 23 out of 2656 respectively. Five percent error was also found during entering the observed values of EQA sample in database of EQAS provider. This means analysis of EQA performance is a helpful tool for the identification of transcription error happened in post analytical phase. Thus, the regular evaluation of EQA provides unique analyses of laboratory results which identify the problems with the performance not always detected by internal quality control activities ${ }^{9}$.

Every analytes analyzed twice to check precision, however this not encouraged because this not exactly done with all patients sample. So, extra attention in EQA sample only may bias and cannot improve overall quality.

\section{CONCLUSION}

The participation of medical laboratory in EQAS program and lucid feedback with statistical index have played key role in quality improvement of laboratory performance. Participation in EQAS along with practice of IQC has been galvanizing GLP by ruling out fallacies of quality performances, progressing annually among EQAS participants and assuring quality service. Evidence found by EQA analysis supports the laboratory professionals to take bold decision to change method or instruments, which is often discouraged by management side.

\section{CONFLICT OF INTEREST}

None Declared

\section{REFERENCES}

1. Plebani M. Appropriateness in programs for continuous quality improvement in clinical laboratories. Clinica chimica acta; international journal of clinical chemistry. 2003;333(2):131-9.

2. Sciacovelli L, Secchiero S, Zardo L, Plebani M. External Quality Assessment Schemes: need for recognized requirements. Clinica chimica acta; international journal of clinical chemistry. 2001;309(2):183-99.

3. Blood Transfusion Safety. Geneva: World Health Organization; External Quality Assessment of transfusion laboratory practice. Guidelines on establishing an EQA scheme in blood group serology;2004, 11-40.

4. Clinical and Laboratory Standard Institute;GP27A2:Using Proficiency Testing to Improve the Clinical Laboratory; Approved Guideline-Second Edition.2007.

5. Chaudhary R, Das SS, Ojha S, Khetan D, Sonker A. The external quality assessment scheme: Five years experience as a participating laboratory. Asian journal of transfusion science. 2010;4(1):28-30.

6. Yadav R, Bhartiya JP, Verma SK, Nandkeoliar MK. External quality assessment scheme (EQAS): Our experience as a participating Laboratory. IJRRMS 2013;3(4): 2-3

7. Olson JD, Preston FE, Nichols WL. External quality assurance in thrombosis and hemostasis: an international perspective. Semin Thromb Hemost. 2007Apr;33(3):220-5.

8. Bejrachandra S, Saipin J, Nathalang O,Siriboonrit U, Rungroung E, Udee S. External quality assessment scheme in red blood cell serology: A 5-year experi-ence in Thailand. Immunohematology. 2006;22:1-5. 
9.Richardson H, Gun-Munro J. Quality improvement through investigation of external quality assess-ment discordant findings. Toronto: Quality Management Program-Laboratory Services; 2005. 
